\title{
A definition of scission points and consequences on some fission distributions
}

\author{
L. Bonneau ${ }^{1, \mathrm{a}}$, P. Quentin ${ }^{1,2}$, and I.N. Mikhailov ${ }^{3,4}$ \\ 1 Theoretical Division, Los Alamos National Laboratory, Los Alamos, New Mexico 87545, USA \\ 2 Centre d'Études Nucléaires de Bordeaux-Gradignan, Université Bordeaux 1, CNRS/IN2P3, Chemin du Solarium, BP. 120, \\ 33175 Gradignan Cedex, France \\ 3 Bogoliubov Laboratory of Theoretical Physics, JINR Dubna, 141980 Dubna, Moscow region, Russia \\ ${ }^{4}$ Centre de Spectrométrie Nucléaire et de Spectrométrie de Masse, CNRS/IN2P3-Université Paris XI, 91406 Orsay-Campus, France
}

\begin{abstract}
A quantitative definition of scission points occurring in a fission process at low energy (spontaneous or induced by low-energy neutron, $\gamma$, charged particle) is proposed. It is based on the concept of a negligible nuclear mutual energy versus its Coulomb counterpart. Practical ways by which one may approximately, albeit mostly in a microscopic fashion (à la Skyrme-Hartree-Fock-BCS), calculate the total energy at scission and their mutual energy parts is presented in a limited yet relevant collective variable space. For a given fragmentation, we present some modeling of the probability for the fissioning system to lie at a given point in the fragment deformation space. From this probability, one deduces the distributions of total fragment-deformation, excitation or Coulomb energies for a given couple of fragments. To yield these distributions some approximations are made and discussed on the sharing of the energy gained in the descent towards scission between kinetic and excitation energies. Some calculational results using the SkM* Skyrme effective interaction and related to the spontaneous fission of the ${ }^{252} \mathrm{Cf}$ nucleus are shown for given fragmentations. As an example, results for the angular momentum distributions in primary fission fragments is compared with available data.
\end{abstract}

\section{Introduction}

The modeling of fission data, such as fragment kinetic and excitation energies, angular momenta, prompt neutron and $\gamma$-emission, requires to clearly separate the fragment formation phase from the fragment acceleration phase in the course of the fission process. It is customary to call scission point the time of the fission process at which this separation occurs. Even though this qualitative definition sounds unambiguous, there is no unique quantitative formulation of this concept. Furthermore the very nature of the scission point is rather to be a distribution of scission configurations, at each of which the above qualitative definition of scission holds.

In this paper we propose a quantitative criterion to characterize the distribution of scission configurations and apply it to the calculation of the distribution of fragment properties. As an example of the latter, we give numerical results for the average fragment angular momenta generated by the "orientation pumping" mechanism $[1,2]$ as a function of the fragment total excitation energy and compare them with experimental data for the spontaneous fission of ${ }^{252} \mathrm{Cf}$ into ${ }^{106} \mathrm{Mo}+{ }^{146} \mathrm{Ba}$.

\section{Scission configurations}

\subsection{Definition of scission}

In the course of a fission process, we may single out a particular time from which the nuclear density in the neck region between the nascent fragments may be considered as

\footnotetext{
${ }^{a}$ Presenting author, e-mail: bonneau@lanl.gov
}

vanishing. In an independent-particle approximation or for a correlated system considering the canonical basis states (diagonalizing the one-body density matrix $\hat{\rho}$ ), after such a time we would ideally expect that the wave function of any nucleon spreads over only one fragment. Therefore, after this event of separation, a given nucleon should then belong to only one fragment and the dynamics of nucleons in one of the fragments $\mathcal{F}_{1}$ become independent of their nuclear interactions with nucleons in the other fragment $\mathcal{F}_{2}$. The dynamics of nucleons in $\mathcal{F}_{1}$ would be therefore governed only by the internal interactions and the external Coulomb potential created by $\mathcal{F}_{2}$ and decreasing with time. This particular point in the fission process is called the scission point.

In practice, however, in Hartree-Fock, Hartree-Fock-BCS (HFBCS) or Hartree-Fock-Bogolyubov (HFB) calculations, the individual wave functions are well defined but there is of course no guarantee that they will belong totally to either fragment, whose particle numbers, moreover, is merely defined by constraining the relevant expectation values. Nevertheless, the one-body density function $\rho(\mathbf{r})$, i.e., the diagonal matrix element in $\mathbf{r}$ of $\hat{\rho}$, is well defined as a whole and its separation in two pieces corresponding to the fission fragments may be judged from purely geometrical arguments. This is actually one of the criteria that Goutte and collaborators retained to define scission in their HFB calculations [3].

Instead, we choose to rely on a dynamical criterion. Assuming that we are able to define nascent or separated fragments, we should try to analyze the forces acting between the two fragments. To this end, we must consider some quantal average over the many nucleon-nucleon interactions. Within an approximate many-body treatment we may evaluate the potential-energy surface from an effective nucleon-nucleon 
interaction for a relevant set of collective dynamical variables. In a simple version of that program we may rather use a phenomenological interaction potential, like the Yukawa-plusexponential (YPE) potential proposed by Krappe, Nix and Sierk [4]. From the knowledge of this potential energy we might derive forces upon taking their derivatives with respect to a collective deformation variable that varies in time much slower than the other variables. A natural scission criterion would then be expressed in terms of the ratio of the norm of the resulting nuclear $\mathbf{F}_{N}$ and Coulomb $\mathbf{F}_{C}$ forces as $\left|\mathbf{F}_{N}\right| /\left|\mathbf{F}_{C}\right|=\eta$, where $\eta$ takes a small value considered as a free parameter. However, a clear separation of the time scales involved in the motion is far from being guaranteed a priori. In view of this and to handle a simple determination of the scission point, we replace the forces in the above ratio with the corresponding nuclear $E_{N}$ and Coulomb $E_{C}$ interaction energies:

$$
\left|\frac{E_{N}}{E_{C}}\right|=\eta .
$$

With this prescription, the scission point occurs whenever the above ratio becomes lower than a critical value of the model parameter $\eta$.

\subsection{Semi-microscopic description of scission configurations}

Since we are interested in spontaneous fission, the initial state of the fissioning nucleus is its ground state, with energy $E_{\mathrm{GS}}$ which is conserved if this nucleus is an isolated system.

After tunneling through the fission barrier, the nucleus appears at points called here exit points exhibiting configurations with a highly-pronounced neck. We consider that a fissioning nucleus can only reach scission configurations that lie beyond (with respect to a global elongation variable) the exit points and, of course, whose energy is less than or equal to $E_{\mathrm{GS}}$. To the scission criterion given by equation (1), we therefore have to add the following condition

$$
E_{\mathrm{sc}} \leqslant E_{\mathrm{GS}}
$$

where $E_{\mathrm{sc}}$ denotes the scission-configuration energy and can be decomposed as

$$
E_{\mathrm{sc}}=E_{C}+E_{N}+\sum_{i=1}^{2} E_{i}^{(\mathrm{sc})} .
$$

In the last term of equation (3), $E_{i}^{(\mathrm{sc})}$ represents the binding energy of the fragment $\mathcal{F}_{i}$.

In microscopic studies of potential-energy surfaces, the potential part of the Hamiltonian $\hat{H}$ of the fissioning nucleus is taken as an effective phenomenological two-body interaction (usually of Skyrme or Gogny type). Although the expectation value of $\hat{H}$ can in principle be calculated at any point of the energy surface, it is difficult to disentangle the four contributions of the right-hand side of equation (3) because $\hat{H}$ is a non-local operator. Furthermore, in order to compute $E_{i}^{(\mathrm{sc})}$, one has to unfold the local densities entering the energy-density functional into sums of two sets of densities localized each in one of the fragments (with some overlap in the neck). This has been done in the case of two spherical nuclei with the Skyrme interaction by Pomorski and Dietrich [5] who showed that the resulting interaction potential is similar to the YPE potential. For that reason and since, expectedly, the conclusions drawn from the final results should not significantly depend on the refinement in the calculation of $E_{N}$, we have decided in the present study to follow a semi-microscopic approach.

We approximate a scission configuration by two axially symmetric fragments with a common symmetry axis. Since the ground-state shapes of the considered nuclei (in the mass region $A \approx 110$ to $A \approx 140$ ) do not exhibit any energetically significant octupole distortions [6], we further assume leftright reflection symmetry for each of them. Altogether, we neglect the mutual polarization effects. In this context, a scission configuration may thus be reasonably well characterized by three shape degrees of freedom: the center-of-mass distance $D_{\text {c.m. }}$ and the elongation of each fragment, expressed as the axial quadrupole moment $Q_{20}^{(i)}$ given by

$$
Q_{20}^{(i)}=\int_{\mathcal{F}_{i}} d^{3} \mathbf{r} \rho(\mathbf{r})\left(2 z^{2}-x^{2}-y^{2}\right) .
$$

In the above expression $\rho(\mathbf{r})$ denotes the total nuclear density (neutron and proton contributions) and the integration is performed over the nuclear volume of fragment $\mathcal{F}_{i}$. The fragment energy $E_{i}$ is therefore assumed to depend only on $Q_{20}^{(i)}$ and is calculated separately for each fragment in the SkyrmeHFBCS approach previously applied to studies involving very large deformations [7-9]. As for the nuclear interaction energy between the two fragments, it has been approximated by its value taken for equivalent coaxial spheroids using the YPE potential (with the parameters of ref. [10]). By equivalent spheroids we mean those having the same elongation $Q_{20}^{(i)}$ and root-mean-square radius as the actual fragments. Similarly the Coulomb interaction energy has been calculated for the equivalent spheroids using the exact analytical expression of ref. [11].

Finally the fragment total kinetic (TKE) and excitation (TXE) energies are calculated at scission as

$$
\begin{gathered}
\mathrm{TKE}=E_{C}+f E_{\mathrm{free}}, \\
\mathrm{TXE}=\sum_{i=1}^{2} E_{\mathrm{def}}^{(i)}+(1-f) E_{\mathrm{free}},
\end{gathered}
$$

where $E_{\text {free }}=E_{\mathrm{GS}}-E_{\mathrm{sc}}, E_{\text {def }}^{(i)}=E_{i}^{(\mathrm{sc})}-E_{i}^{(\mathrm{GS})}\left(E_{i}^{(\mathrm{GS})}\right.$ being the ground-state binding energy of $\mathcal{F}_{i}$ ) and $f$ is a model parameter controlling the partitioning of available energy at scission $E_{\text {free }}$ between the prescission kinetic energy $f E_{\text {free }}$ and the total intrinsic fragment excitation energy $(1-f) E_{\text {free }}$.

\section{Distributions of fragment properties at scission}

The distribution over the scission configurations should result from a relevant quantal calculation for the collective dynamics. Upon considering a given TXE-value, out of the three model collective variables, two are fixed by equations (1) and (6). The remaining variable is called $s$ and defined below. To mock up 


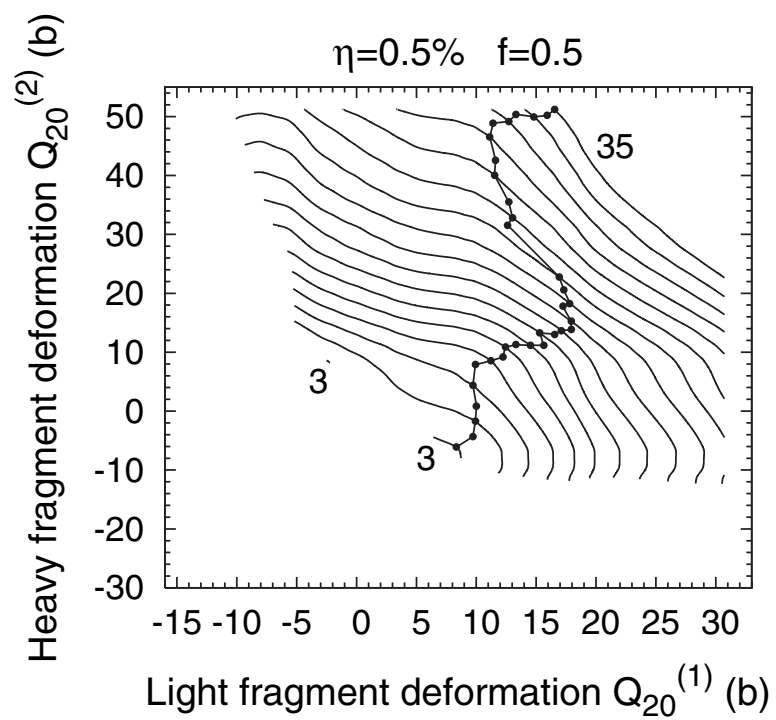

Fig. 1. Contour maps of TXE at scission from 3 (lowest value) to $35 \mathrm{MeV}$ for ${ }^{106} \mathrm{Mo}+{ }^{146} \mathrm{Ba}$ scission configurations obtained with $\eta=0.5 \%$ and $f=0.5$. The dots indicate the location on each level contour of the most-probable scission configuration (maximal value of $w$ ).

the above distribution in this exploratory study, we assume a weight of the form

$$
w(s)=e^{-E_{\mathrm{sc}}(s) / \Theta} .
$$

Even though the simulation of the spreading among scission configurations seems to refer to the approach of Wilkins and collaborators [12], it is different in spirit since we invoke here a purely quantal fluctuation, which yields a Gaussian distribution when, at a given TXE, $E_{\mathrm{sc}}(s)$ varies quadratically around its minimum.

To determine completely the distribution probability for a fixed value of TXE, we need to choose the values of $\eta, f$ and $\Theta$. On the one hand, the scission configurations satisfying equation (1) must be such that the particle transfer between both fragments ceased, which corresponds to a vanishing neck radius. This imposes an upper limit on $\eta$. On the other hand, given the uncertainties on the Coulomb and nuclear interaction energies approximated as above mentioned, it is safer to discard $\eta$-values leading to very small nuclear interaction energies (below, say, $0.5 \mathrm{MeV}$ or so). We therefore choose $\eta=0.5 \%$. As for $f$, we choose three values: the lower limit 0 , the upper limit 1 and an intermediate value, 0.5. Finally, we assume that the elongation degree of freedom is much slower than the single-particle ones and that $\Theta$ represents a typical collective excitation energy in a mode involving the variable $s$ yielding a characteristic time for this motion much shorter than a typical time for the descent from saddle to scission. Therefore $\Theta$ must be much larger than a couple of $\mathrm{MeV}$ [13] and we choose here $\Theta=15 \mathrm{MeV}$.

To obtain the mean value $\bar{X}$ of an observable $X$ we average $X$ over the solutions of equations (1) and (6) for a fixed TXE-value by a curvilinear integration on the contour $C_{\text {TXE }}$

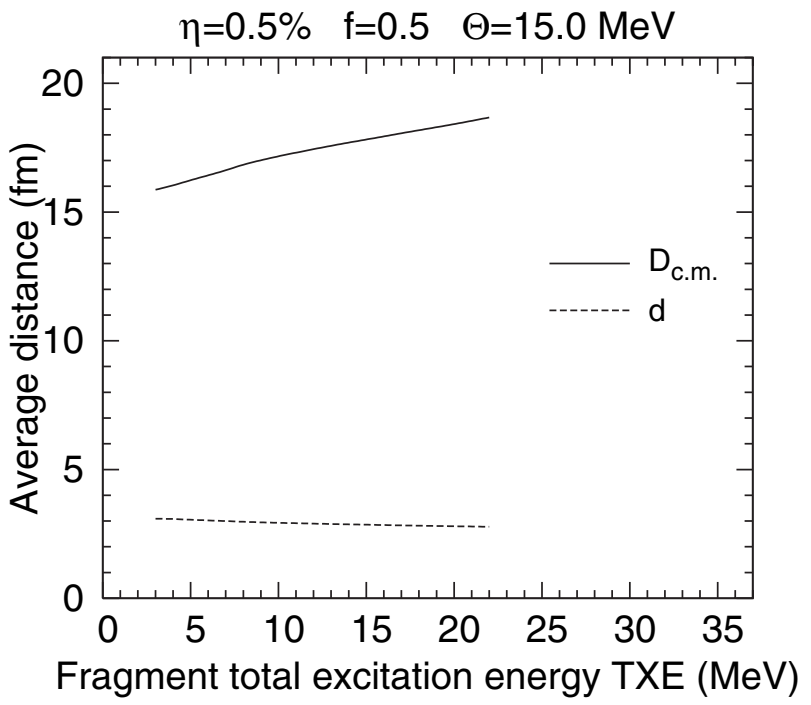

Fig. 2. Average center-of-mass distance $D_{\text {c.m. }}$ and tip distance $d$ as a function of TXE for ${ }^{106} \mathrm{Mo}+{ }^{146} \mathrm{Ba}$ scission configurations obtained with $\eta=0.5 \%, f=0.5$ and $\Theta=15 \mathrm{MeV}$.

connecting the scission configurations

$$
\bar{X}=\frac{1}{\mathcal{N}} \int_{\mathcal{C}_{\mathrm{TXE}}} d s X\left(Q_{20}^{(1)}(s), Q_{20}^{(2)}(s)\right) w(s),
$$

with the normalization factor $\mathcal{N}=\int_{C_{\mathrm{TXE}}} d s w(s)$ and where $s$ is the curvilinear abscissa along $C_{\mathrm{TXE}}$. An example of such contours in the $\left(Q_{20}^{(1)}, Q_{20}^{(2)}\right)$ plane is shown in figure 1 for $\eta=0.5 \%$ and $f=0.5$. To define specifically the curvilinear abscissa $s$ we need to redefine our choice of collective variables so as to ensure their consistency (same units, comparable range of variations). More importantly we need to choose a metric in the resulting 3D space. Without undertaking definite mechanical studies implying the relevant potential energy and inertia parameters, it is impossible to avoid making rather arbitrary choices for both the definition of the collective variables and the associated metric. The three collective variables which we consider are the three components of the total quadrupole moment $Q_{20}^{(\text {tot })}$, namely $Q_{20}^{(1)}, Q_{20}^{(2)}$ and $Q_{D}$, where the latter is defined by

$$
Q_{D}=Q_{20}^{(\mathrm{tot})}-Q_{20}^{(1)}-Q_{20}^{(2)}=\frac{2 A_{1} A_{2}}{A_{1}+A_{2}} D_{\mathrm{c} . \mathrm{m} .}^{2}
$$

In equation (9), $A_{i}$ denotes the nucleon number of the fragment $i$. We find it appropriate not to single out one dynamical variable over the others and adopt the Cartesian metric $d s^{2}=$ $\left(d Q_{20}^{(1)}\right)^{2}+\left(d Q_{20}^{(2)}\right)^{2}+\left(d Q_{D}\right)^{2}$.

As can be seen in figure 2 representing the average tip distance $d$ (dashed line) and the center-of-mass distance $D_{\text {c.m. }}$. (solid line) obtained with the typical values $\eta=0.5, f=0.5$ and $\Theta=15 \mathrm{MeV}$, the former remains constant as TXE varies whereas $D_{\text {c.m. }}$ increases with TXE. With the above selected parameter values, we find a $d$-value of about $3 \mathrm{fm}$, much larger than the one considered by Wilkins et al. [12] $(d=1.4 \mathrm{fm})$ or in ref. [14]. 


\section{Application to fission-fragment spin generated by orientation pumping}

Fission fragments resulting from the fission at low energy (neutron or light charged particle induced fission) or even zero energy (as in spontaneous fission) end up with angular momenta widely distributed in a range going from typically a few to about ten units of $\hbar$. This result has been obtained long ago and further confirmed in a variety of different experimental situations (for reviews and comprehensive references see, e.g., [15-17]). Various theoretical attempts to describe the generation of angular momentum in fission fragments have dwelled in the conception of an excitation at scission of some collective modes capable of producing angular momenta. The rather elusive character of such a specific scission point renders this type of approaches somewhat contingent upon specific assumptions on the collective dynamics at this stage of the fission process. Here we consider the quantum-mechanical "orientation-pumping" mechanism proposed in refs. [1,2] and discard other sources of spin in order to assess the ability of this mechanism alone to reproduce the order of magnitude and the trend of the average fragment spins as a function of TXE. A review of this mechanism can be found in ref. [13]. Here, we recall only the main expression for the expectation value of the square of the angular momentum operator, $\hat{\mathbf{J}}^{2}$, in the state of the fissioning nucleus at scission, derived under the assumption of a pure-rotor distribution of angular-momentum projected energies of each fragment [1]

$$
\left\langle J^{2}\right\rangle=\left(\frac{1}{\left\langle J_{1}^{2}\right\rangle_{\text {intr }}}+\frac{1}{\left\langle J_{2}^{2}\right\rangle_{\text {intr }}}\right)^{-1},
$$

where $\left\langle J_{i}^{2}\right\rangle_{\text {intr }}$ stands for the intrinsic expectation value of the $\hat{\mathbf{J}}^{2}$ operator evaluated for the wave function of the fragment $i$. If one of the fragment is spherical, then this formula yields vanishing fragment spins. Therefore this expression applies only to deformed fragments.

As can be seen in figure 3 , the average fragment angular momentum $J_{\mathrm{f}}$ steadily increases with TXE, hence with the prompt neutron multiplicity $\bar{v}$ which varies approximately linearly with TXE. This is in agreement with the experimental data of ref. [18], and so is the order of magnitude calculated here (a few $\hbar$ units).

\section{Conclusions and perspectives}

The description of scission configurations proposed here allows for a study of the prescission kinetic energy contribution to TKE and its influence on other fission quantities, like the fragment angular momenta. Calculated in the orientationpumping mechanism alone, the average fragment spins are found to be compatible with the available experimental data. In a near future we should give up the good-rotor approximation and perform the angular-momentum projection. In addition the scission configurations should be determined in a fully microscopic picture incorporating the mutual polarization effects.

We gratefully acknowledge many enlightening discussions with Pr. F. Gönnenwein. One of the authors (Ph.Q.) thanks the Theoretical

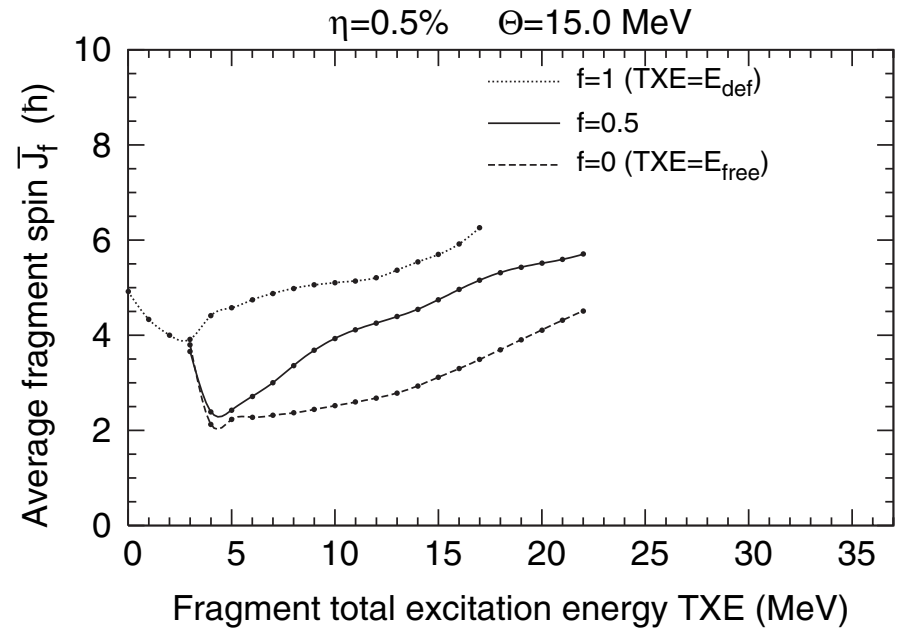

Fig. 3. Variation of the average angular momentum in units of $\hbar$ at scission with the total excitation energy for the ${ }^{106} \mathrm{Mo}+{ }^{146} \mathrm{Ba}$ fragmentation for $\eta=0.5 \%$.

Division at LANL for the excellent working conditions extended to him during numerous visits. Another (I.N.M.) acknowledges a valuable support from the JINR-IN2P3 agreement (collaboration 03-61). This research has been supported by the US Department of Energy under contract W-7405-ENG-36, the IN2P3/CNRS and the JINR.

\section{References}

1. I.N. Mikhailov, P. Quentin, Phys. Lett. B 462, 7 (1999).

2. I.N. Mikhailov, P. Quentin, Ch. Briançon, Yad. Fiz. 64, 1185 (2001); Phys. At. Nucl. 64, 1110 (2001).

3. H. Goutte, J.-F. Berger, D. Gogny, Int. J. Mod. Phys. E 15, 292 (2006).

4. H.J. Krappe, J.R. Nix, A.J. Sierk, Phys. Rev. C 20, 992 (1979).

5. K. Pomorski, K. Dietrich, Z. Phys. A 295, 355 (1980).

6. P. Möller, J.R. Nix, W.D. Myers, W.J. Swiatecki, At. Data Nucl. Data Tables 59, 185 (1995).

7. L. Bonneau, P. Quentin, D. Samsœn, Eur. Phys. J. A 21, 391 (2004).

8. L. Bonneau, P. Quentin, Phys. Rev. C 72, 014311 (2005).

9. L. Bonneau, P. Quentin, Proceedings of the 3rd International Workshop on Nuclear Fission and Fission-Product Spectroscopy edited by H. Goutte, H. Faust, G. Fioni, D. Goutte (AIP, Melville, NY, 2005), p. 77.

10. A.J. Sierk, Phys. Rev. C 33, 2039 (1986).

11. P. Quentin, J. Physique (Paris) 30, 497 (1969).

12. B.D. Wilkins, E.P. Steinberg, R.R. Chasman, Phys. Rev. C 14, 1832 (1976).

13. L. Bonneau, P. Quentin, I.N. Mikhailov (accepted by Phys. Rev. C)

14. Ş. Mişicu, P. Quentin, Eur. Phys. J. A 6, 399 (1999).

15. R. Vandenbosch, J.R. Huizenga, Nuclear Fission (Academic Press, New York, 1973), Ch. XIII.

16. D. de Frenne, The Nuclear Fission Process edited by C. Wagemans (CRC Press, Boca Raton, 1991), Ch. 9.

17. I. Ahmad and W.R. Phillips, Rep. Prog. Phys. 58, 1415 (1995).

18. G.S. Popecko et al., Proc. of the 2nd Int. Conf. on Fission and Properties of Neutron-Rich Nuclei (World Scientific, Singapore, 1998), p. 645; G.M. Ter Akopian et al., Proc. of the 3rd Int. Conf. on Fission and Properties of Neutron-Rich Nuclei (World Scientific, Singapore, 1999), p. 98. 